\title{
polyClustR: defining communities of reconciled cancer subtypes with biological and prognostic significance
}

\author{
Katherine Eason ${ }^{1}$, Gift Nyamundanda ${ }^{1,2}$ and Anguraj Sadanandam ${ }^{1,2^{*}}$ (D)
}

\begin{abstract}
Background: To ensure cancer patients are stratified towards treatments that are optimally beneficial, it is a priority to define robust molecular subtypes using clustering methods applied to high-dimensional biological data. If each of these methods produces different numbers of clusters for the same data, it is difficult to achieve an optimal solution. Here, we introduce "polyClustR", a tool that reconciles clusters identified by different methods into subtype "communities" using a hypergeometric test or a measure of relative proportion of common samples.

Results: The polyClustR pipeline was initially tested using a breast cancer dataset to demonstrate how results are compatible with and add to the understanding of this well-characterised cancer. Two uveal melanoma datasets were then utilised to identify and validate novel subtype communities with significant metastasis-free prognostic differences and associations with known chromosomal aberrations.

Conclusion: We demonstrate the value of the polyClustR approach of applying multiple consensus clustering algorithms and systematically reconciling the results in identifying novel subtype communities of two cancer types, which nevertheless are compatible with established understanding of these diseases. An R implementation of the pipeline is available at: https://github.com/syspremed/polyClustR
\end{abstract}

Keywords: Clustering methods, Subtype community, Reconciliation methods, Network analysis, Hypergeometric test, Breast cancer, Uveal melanoma, Hierarchical clustering, K-means clustering, Non-negative matrix factorization

\section{Background}

Recently, advances in omics technologies have lead to large volumes of data being collected on molecular profiles, including gene expression, in various cancers. Cancers of all types exhibit inter-tumoral (between patient) heterogeneity that can be quantified in part by gene expression. This heterogeneity can help explain the differential prognosis in cancer patients treated with the same therapies. A well-established example is the specific efficacy of trastuzumab (Herceptin) in HER2-positive breast cancer [1]. Previously, we suggested potential differential cetuximab (anti-EGFR therapy) responses in colorectal cancer (CRC) subtypes that we defined previously [2]. More recently, trials of oxaliplatin in Stage II and III

\footnotetext{
* Correspondence: Anguraj.Sadanandam@icr.ac.uk

'Division of Molecular Pathology, The Institute of Cancer Research (ICR), London, UK

${ }^{2}$ Centre for Molecular Pathology, Royal Marsden Hospital (RMH), London, UK
}

CRC found that its effectiveness may be limited to one of these subtypes $[2,3]$. In pancreatic cancer, we observed a relatively increased response to gemcitabine in quasi-mesenchymal (QM) subtype cell lines compared to classical subtype cell lines [4]. This result corroborates with the finding by Mofitt et al., that patients with the basal-like pancreatic cancer subtype (equivalent to our QM subtype) have improved response to adjuvant therapy compared to those with the classical pancreatic cancer subtype [5]. Similarly, we showed potential subtype-specific therapies using a panel of breast cancer cell lines and drug response analysis [6]. Nevertheless, for accurate prediction of therapy responses, the challenge lies in defining robust and clinically relevant subtypes.

In breast cancer, where current opinion lies with the existence of 5 intrinsic gene expression subtypes (basal, HER2/ERBB2, luminal A, luminal B, and normal-like), studies have variously reported a number of gene 
expression subtypes ranging between 4 [7] and 10 [8]. While multiple factors are involved in this apparent discrepancy in defining a number of cancer subtypes, the clustering methodologies employed can significantly contribute to this difference. There are various clustering algorithms that are regularly employed for this purpose, and each has its own strengths according to the underlying structure of the data it is applied to. As clustering algorithms have a huge range of potential applications, selection of the appropriate algorithm to use in any given situation can be difficult. At the same time, the need for the user to inspect the results of each algorithm over a range of numbers of clusters $(k)$ and select the optimal solution are often subjective. This situation has been improved by the adoption of various consensus clustering techniques, which allow for visual and quantitative examination of multiple re-runs of the same algorithm so the effects of random starting points can be taken into consideration.

Nevertheless, the use of consensus clustering does not mitigate the effect that algorithm choice has on the clustering solution. The application of different consensus clustering algorithms leads to different numbers of subtypes (numbers of clusters, $k$ ), and hence, defining the optimal number of clusters is often challenging. This is due to various factors in the design of the algorithm: whether it is 'greedy', that is, if it makes the locally optimal choice at each individual stage at the possible expense of finding a global optimum; whether cluster centroids must be located at data points; how iterative algorithms evaluate their convergence to a solution; the "shape" of the discovered clusters; and the metric used to measure sample similarity are some examples [9]. The high dimension of biological data can also demand unreasonable computational time and create a large search domain for the optimal solution. This makes the use of a single algorithm to cluster gene expression profiles, as is often done in subtyping studies, risky. In addition, the clusters found may well be valid, but information about either larger stratification of the data or small but distinct sub-subtypes of low frequency may be lost [10]. It is for this reason that finding methods of reconciling optimal clustering solutions identified by different algorithms is necessary. Cluster reconciliation not only validates the clusters from different algorithms - it can also reveal in greater detail the structure in the data on the macro and the micro scale, from broad classifications resulting from a handful of important functional groups, to rarer and less well-defined sub-subtypes [10, 11].

Here, we demonstrate how to identify optimal solutions and define subtype "communities" by reconciling clusters identified from three different consensus clustering methods - hierarchical clustering (HC) [12, 13], k-means (KM) [14], and non-negative matrix factorization (NMF)
[15]. The clusters were further reconciled using at least two approaches. The first, a hypergeometric test to determine the probability that two clusters share the same samples by chance, was previously used to successfully reconcile subtypes of CRC found via clustering in two studies which found three and five optimal subtypes, respectively $[2,10,16]$. It was determined via this analysis that the three subtypes could be appropriately divided into the five sub-subtypes. When four further studies into CRC were published, finding between 3 and 6 optimal clusters [17-20], the Jaccard index was applied to help understand the relationships between these solutions and find "consensus molecular subtypes" (CMS) [11]. The second and a new reconciliation measure used here - calculating the relative proportion of samples in a smaller cluster present in a larger one (termed PMI) - differs from measures of cluster similarity such as the Jaccard index in order to give sub-subtypes a high score, even if they are much smaller than a larger cluster (see Methods section).

All the above reconciliation methods are part of our new framework or package called "polyClustR". The framework is flexible that other methods can be included any time. Here, we demonstrate how our new pipeline can be used to identify breast cancer gene expression "subtype communities" and to compare with existing intrinsic subtypes [7]. Moreover, we have applied this to uveal melanoma gene expression profiles to define novel gene expression "subtype communities" with different prognosis and chromosomal aberrations associated with them.

\section{Methods}

\section{Datasets}

The breast cancer dataset (Chin, et al.) [21] consists of 118 gene expression profiles of 12,703 genes generated from frozen resected samples. Patients in this cohort were mostly early-stage, and were a mixture of node- and ER-positive and -negative. The discovery uveal melanoma dataset (GSE22138 [22]) consists of gene expression profiles of 42,346 genes for 63 untreated patients, chromosome 3 monosomy status and follow-up metastasis-free survival information. The validation dataset (GSE44295 [23]) contains 58 gene expression profiles (only the tumor samples were considered) for 24,526 genes from enucleation specimens, with metastasis-free survival information [24].

\section{Finding the optimal number of clusters}

It is not optimal for each of the above clustering methods to find a local solution which depends on the initial conditions, rather than a robust clustering that is stable over various input parameters. To address this, we used consensus clustering approaches that repeat several iterations of the same algorithm using different random starting points, and can also perform the 
clustering over different subsets of samples. Consensus clustering for each algorithm was performed over a range of $k$-values from 2 to 10 and over multiple subsets of the data $(80 \%$ of samples randomly selected for each of 100 runs). The results of the consensus clustering were then inspected in order to determine the optimal $k$. Determining the optimal $k$ from visual inspection alone is subjective, and so quantification of the consensus clustering is required. Here, the cophenetic correlation coefficient [25] and the silhouette width [26] were used to score each clustering. As there is no "gold standard" method for the selection of the value of $k$, the tool user is prompted to choose the number of clusters they wish to use for each algorithm downstream reconciliation based on this output. This allows for flexibility based on the goals of the analysis, as the user can choose a high value of $k$ if the objective is to, perhaps, identify novel subgroups of a well-characterised cancer, or to choose a low value of $k$ if this solution is more robust.

\section{Hypergeometric test}

Previous work has used the hypergeometric test to determine if different algorithms' subtypes correspond to one another [10]. In this pipeline, comparisons can be made between any number of clustering algorithms. The hypergeometric test-based false discovery rate (FDR)-adjusted $p$-value indicating the significance of the size of the overlap between two clusters was used.

\section{Proportion of maximum intersection (PMI)}

We introduce the following proportion of maximum intersection, PMI; for clusters $A$ and $B$, of arbitrary size:

$$
P M I=\frac{(A \cap B)}{\min \{|A|,|B|\}}
$$

The measure above gives the proportion of samples shared between two clusters out of the maximum possible samples shared (that is, the number of samples in the smaller cluster). If, for example, $A$ contains more samples than $B$, PMI is 0 when none of the samples of $B$ are in $A$ and 1 when all of the samples of $B$ are in $A$. This measure has an advantage over alternatives such as the Jaccard index (used in our previous publication [11]) in this context, for the reason that in having different values of $k$ clusters from different algorithms will inherently be of different sizes. If the same number of clusters were being compared between algorithms then Jaccard index would give a good measure of cluster overlap. Using our alternative metric gives the same weight to clusters of the same size which are directly analogous between algorithms, as it does to clusters identified via one algorithm which are sub-subtypes of a cluster found by a different algorithm.

\section{Network community detection}

The FDR values produced by the hypergeometric tests and the PMI scores can be represented as edge weights in an undirected network, where vertices are clusters as discovered by the various algorithms. This network can be subjected to community detection, where groups of well-connected vertices (clusters) are identified. In the label propagation method [27] each vertex is initialized with a different label, before then being assigned the label that is most common amongst its direct neighbors. This process continues iteratively until convergence. A variant of the label propagation algorithm [27], which takes into account the weight of edges connecting the vertices, is used here.

\section{Statistical analysis}

FDR values for enrichment of gene sets were reported as calculated by the Broad Institute's GSEA software [28]. $P$-values generated by hypergeometric tests were FDR-corrected for multiple testing. Kaplan-Meier analysis was used to assess survival and the statistical analysis were from log-rank test. Prediction analysis of microarrays (PAM) analysis to generate centroids and assign subtypes using Pearson correlation and gene expression data was done as previously described [11].

\section{Software}

Code for hierarchical and k-means consensus clustering was adapted from the ConsensusClusterPlus v1.36.0 [29] $\mathrm{R}$ package. NMF was performed via the $n m f \mathrm{v} 0.20 .6 \mathrm{R}$ package [30]. The igraph $\mathrm{R}$ package v1.0.1 [31] was used for plotting networks and community detection. Silhouette width was calculated and plotted using the silhouette function from the R package cluster v2.0.4 [32]. Survival analysis was performed using the survival v2.39-5 R package [33]. The pipeline described in this paper is publicly available on GitHub at https://github.com/syspremed/polyClustR. Clustering parameters for the pipeline are: Consensus resamplings: 100; Proportion of items sampled per subsample: 0.8; Clustering distance: Euclidean; Heirarchical linkage method for subsampling: Average; Heirarchical linkage method for consensus matrix: Average.

\section{Results and discussion}

\section{An overview of the tool}

Our reconciliation method (Fig. 1) uses a matrix of preprocessed and normalized gene expression (or any other similar data) and performs the following: a) applies different consenusus clustering methods (including NMF, $\mathrm{HC}$ and $\mathrm{KM}$ ) and uses statistical scores (specific to each method described below) for each clustering to allow the user to choose the optimal number of clusters; and b) reconciles the results from different clustering methods and identifies a consensus solution by creating network 


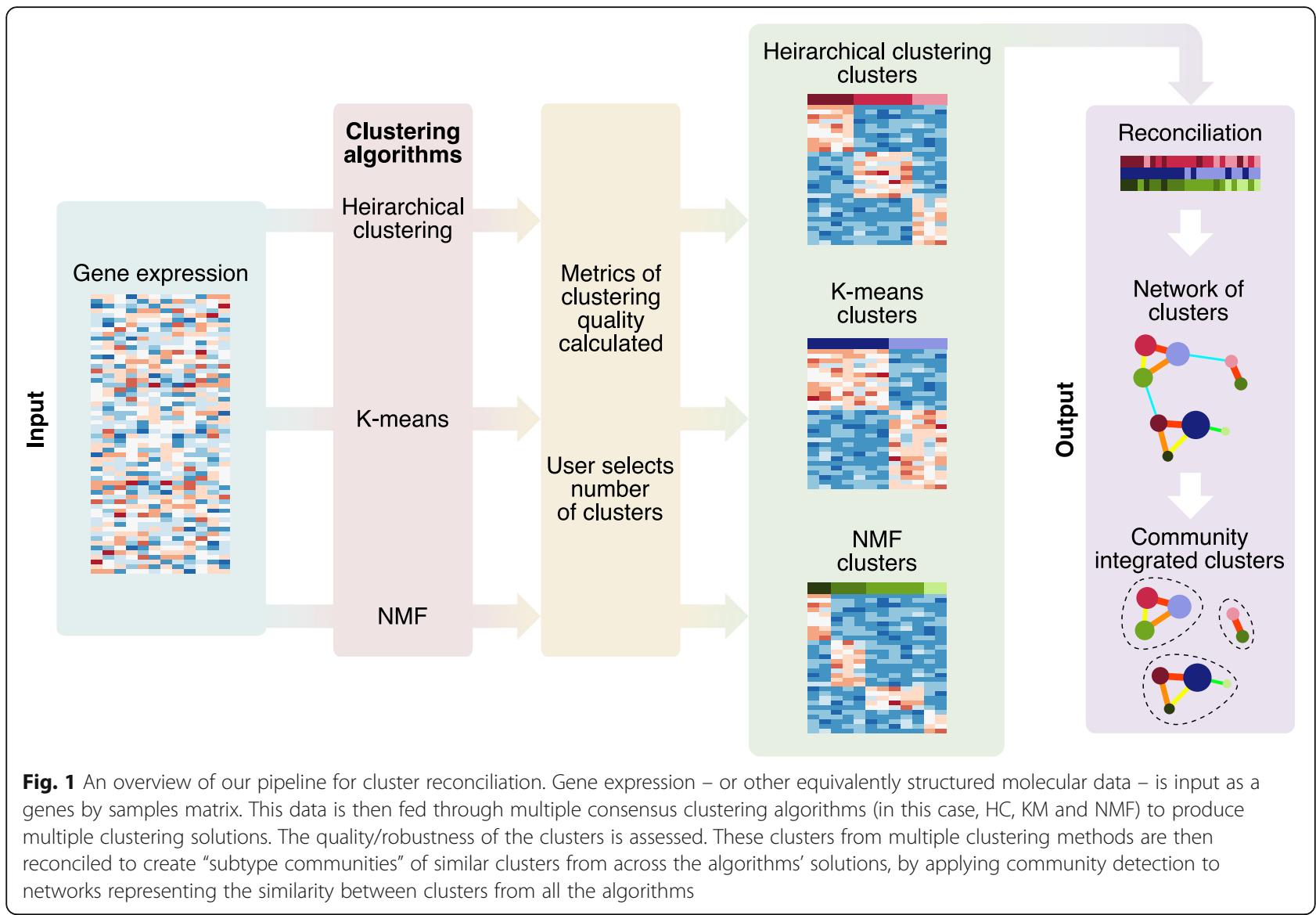

of clusters that defines "communities" of integrated subtypes using methods such as the hypergeometric test and PMI. We then identify the optimal "communities" with highest average silhouette width [26] and compare this reconciliation to known subtypes, if they exist, for that set of samples. To illustrate this, we used published gene expression profiles from breast cancer and uveal melanoma as examples.

\section{Application to reconcile breast cancer "subtype communities" with intrinsic subtypes}

Breast cancer subtypes defined by multiple clustering methods

For this purpose, we used breast tumor gene expression data ( $n=118$; Chin data set) from a published study [21]. Details of initial clustering of this dataset and selection of $k$ clusters for each algorithm are provided in Additional file 1: Figure S1A-D. Initially, we applied NMF to the 2258 most highly variable genes from this Chin data set as selected by standard deviation (SD >0.8). We identified highest cophenetic correlation coefficient of 0.9997 for NMF $k_{N M F}=2$ followed by 0.9962 at $k_{\mathrm{NMF}}=6$. Silhouette width also showed peaks at $k_{\mathrm{NMF}}$ at 2 and 6 (Additional file 1: Figure S1A-B). In this work, as the downstream reconciliation methods will pool any similar clusters together, we choose the higher value of $k$ where the cophenetic correlation coefficient and silhouette width are approximately equal between different values of $k$. In addition, we assessed the consensus matrix for each $k$ and evaluated the number of outlier samples (less the better) in each cluster. This allows us to retain the finer stratification of the initial clustering, while simultaneously assembling information about broader biological characteristics from the subsequent reconciliation. As such, we chose $k_{\mathrm{NMF}}=6$, and named the clusters breast cancer (b)NMF1 to 6. Overall, known subtypes of these samples [21] were significantly associated with these clusters (Fisher's exact test; $p<0.001$ ). Specifically, the clusters bNMF1, bNMF3 and bNMF4 were significantly associated with luminal A, basal and luminal B, respectively (hypergeometric test; false discovery rate; $F D R<0.01$ ) (Fig. 2a). The basal subtype was also border-line significantly associated $(F D R=0.2)$ with bNMF5, suggesting the existence of a sub-subtype of basal breast cancer that was not identified earlier when subtypes for this dataset were predicted by correlation with intrinsic subtype signatures [34] [7]. bNMF2 and bNMF6 were not significantly associated with any of the published subtypes. Gene set enrichment analysis (GSEA) of these unidentified subtypes revealed associations with metaplastic breast cancer (bNMF2, 

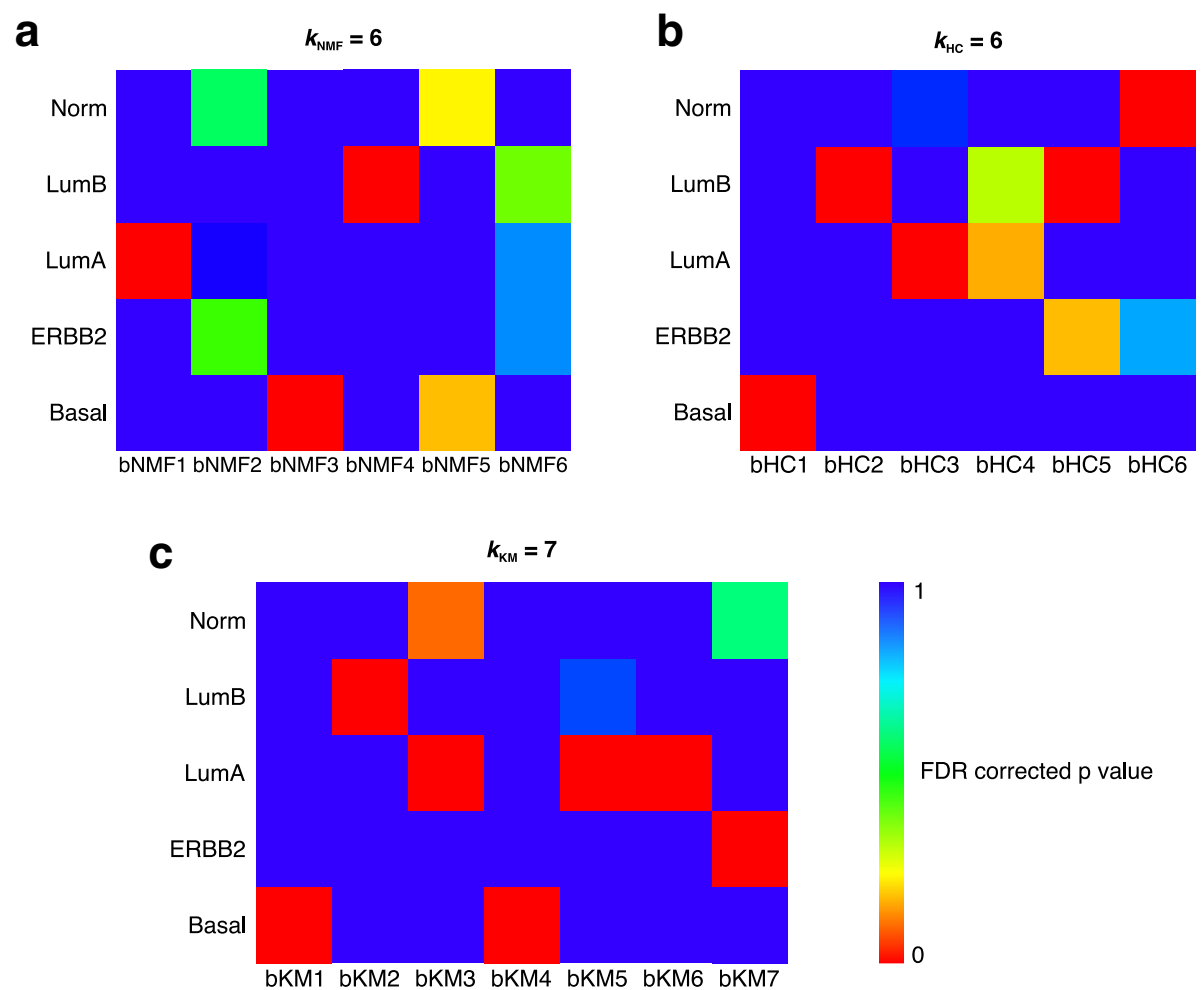

Fig. 2 Breast cancer subtypes and their association with intrinsic subtypes - application of polyClustR. a-c Heatmaps of similarity of each set of clusters generated by consensus a NMF, b HC and $\mathbf{c}$ KM to the known breast cancer subtypes of each sample using 118 breast cancer samples from the published Chin dataset [21] is shown. A hypergeometric test was used to test the significance of overlap between the clusters and the known subtypes. bNMF, bHC and bKM represent NMF, HC and KM breast cancer subtypes, respectively. Norm - normal-like, LumA - luminal A and LumB - luminal B subtypes. FDR - false discovery rate

$F D R<0.01)$ and with $17 \mathrm{q} 21-\mathrm{q} 25$ amplicon gene sets (bNMF6, FDR $<0.1$ ) (Additional file 2: Figure S2A-B). Overall, application of NMF to the Chin data set identified clusters that partially overlapped with published subtypes, and others with interesting breast cancer biology.

Since NMF identified extra subtypes in the Chin data set, we applied two additional clustering methods - consensus hierarchical clustering (HC) and k-means (KM). When we applied consensus hierarchical clustering to the same data, $k_{\mathrm{HC}}=2$ and $k_{\mathrm{HC}}=6$ had the highest silhouette widths. (Additional file 1: Figure S1A and C). The cophenetic coefficient after $k_{\mathrm{HC}}=6$ does not increase significantly and the consensus plot showed well-defined clusters (Additional file 1: Figure S1A and C). Hence, we chose six HC clusters (as it reduces the heterogeneity and further downstream reconciliation will group any similar clusters together). The clusters from $\mathrm{HC}$ for breast cancer data were defined as breast cancer (b)HC. As with the NMF clusters, these clusters were significantly associated with the known subtypes of these samples (Fisher's exact test; FDR $<0.001$ ). The bHC1, bHC3 and bHC6 clusters were significantly (hypergeometric test; $F D R<0.01)$ associated with basal, luminal $\mathrm{A}$ and normal-like subtypes, respectively (Fig. 2b). Both bHC2 and bHC5 were significantly $(F D R<0.01)$ associated with the luminal B subtype. bHC4 was marginally significantly associated with the luminal A subtype, and bHC5 with the ERBB2 (HER2) subtype, with less significance $(F D R<0.2$; Fig. 2b).

Additionally, we applied consensus KM clustering to the Chin data set. While both the cophenetic coefficient and silhouette width showed highest peaks at $k_{\mathrm{KM}}=3$ and 4 (after $\left.k_{\mathrm{KM}}=2\right)$, we observed that consensus clustering at these $k_{\mathrm{KM}}$ values did not show clear consensus clusters. There were not large differences in cophenetic coefficient, silhouette width and consensus clusters at $k_{\mathrm{KM}}$ between 4 and 7 (Additional file 1: Figure $\mathrm{S} 1 \mathrm{~A}$ and $\mathrm{D})$. Hence, we chose $k_{\mathrm{KM}}=7$ as an optimal cluster. All of these KM clusters (defined as breast cancer (b)KM were significantly or marginally significantly associated with known breast cancer subtypes (Fig. 2c; Fisher's exact test; $p<0.001$ ), unlike the NMF and HC clusters. Specifically, bKM1 and bKM4 were associated with basal, bKM2 with luminal B and bKM3, bKM5 and bKM6 with luminal A (hypergeometric test; $F D R<0.01$ ). bKM7 was significantly associated with the ERBB2 subtype, which was not highly significant with any NMF or 
HC clusters. bKM3 was marginally associated with the normal-like subtype $(F D R=0.08)$. Direct comparison of the two basal clusters through GSEA revealed enrichment of multiple gene sets associated with invasive breast cancer, immunity and cytokines (Additional file 2: Figure S2C-F; with FDR $<0.2$ ). This clearly suggests that different clustering algorithms have the inherent capacity to identify distinct clusters. Here, KM has identified clusters with more significant association to all the published subtypes.

\section{Identification of breast cancer "subtype communities"}

The existence of multiple clustering solutions defined by different algorithms poses the question of what number of clusters is optimal, and how they reconcile between different methods. To address these questions, we chose two different reconciliation methods - hypergeometric test and PMI. The results from each of the reconciliation methods are discussed below.

Previously, we have used the hypergeometric test to assess enrichment of samples between two CRC classifications (including ours) as a means of reconciling subtypes [10]. Similarly, we have used this analysis here to reconcile breast cancer clusters between the three different (NMF, HC and KM) algorithms utilized above. Subsequently, in order to group those clusters with significant similarity into "subtype communities", we performed network community detection by applying weighted label propagation method (using FDR values as edge weights) [27]. As a result, we observed six "subtype communities" (groups of clusters; bHYP1-6) based on this analysis (Fig. 3a).

There was significant association with the known subtypes and these communities (Fisher's exact test; $p<0.001)$. We observed that five communities were primarily and significantly (hypergeometric test; FDR $<0.05)$ associated with published breast cancer subtypes - bHYP3 and bHYP4 with luminal A, bHYP2 with luminal B, and bHYP1 and bHYP6 with basal (Fig. 3a and Additional file 3: Figure S3A). Four of the communities (bHYP1-4) contained clusters from all three clustering algorithms (Fig. 3a). Interestingly, each of the luminal A and basal subtypes were split into two communities. One basal community (bHYP6) contained the immune-enriched bKM4 cluster. One of the luminal A communities (bHYP3) contained a number of samples from the ERBB2 subtype in a cluster that was enriched for a metaplastic breast cancer signature (bNMF2; Fig. 3a and Additional file 3: Figure S3A), while the other (bHYP4) contained some luminal B samples in the 17q21-q25 amplicon-enriched cluster (bNMF6; Fig. $3 \mathrm{a}$ and Additional file 3: Figure S3A). Finally, there was a community (bHYP5) with mixture of normal-like and ERBB2 subtype samples. This community was the most mixed in terms of intrinsic subtypes. Overall,

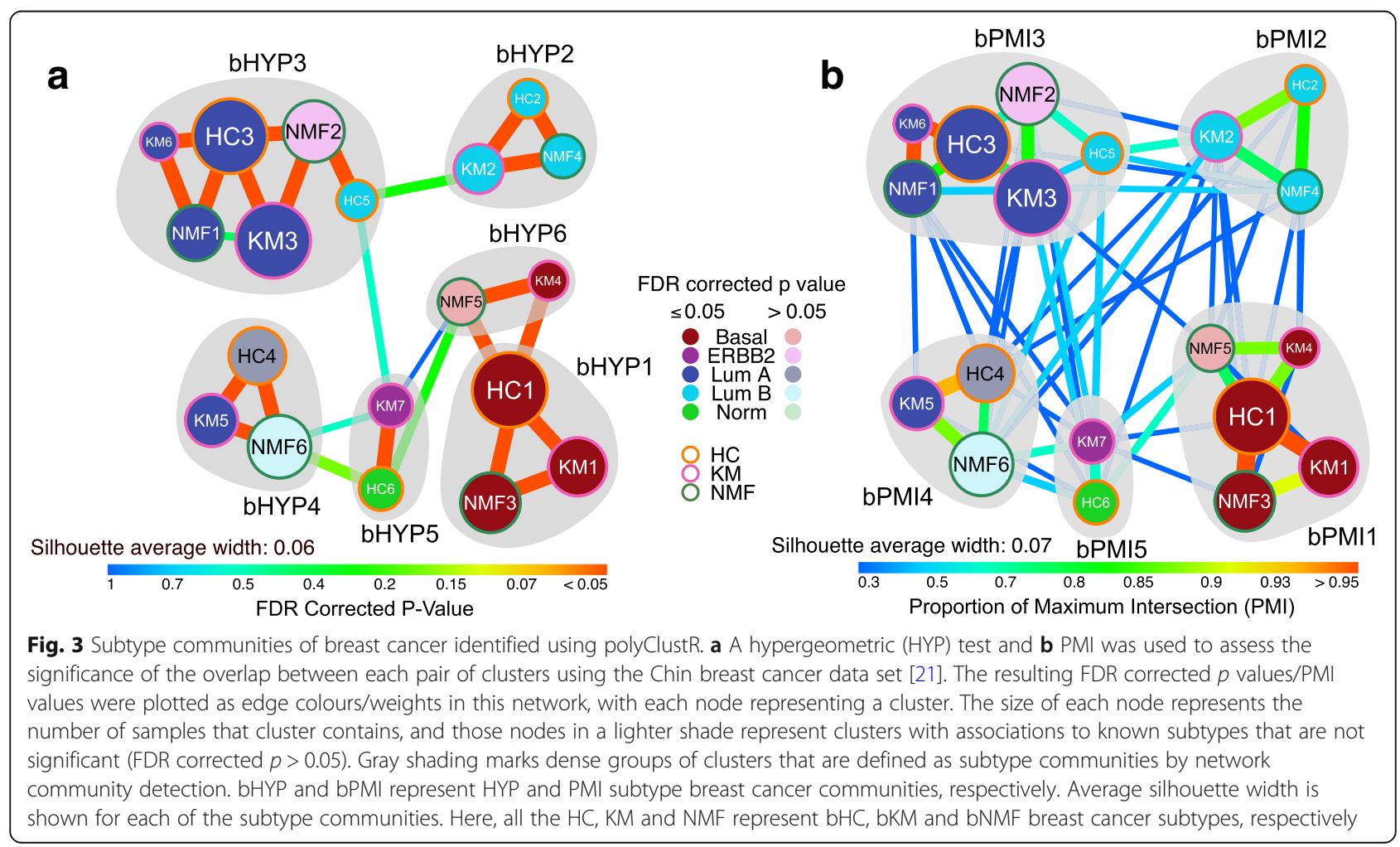


hypergeometric test-based reconciliation expanded the breast cancer subtypes to 6 communities.

Our PMI method is similar to the Jaccard analysis that we used recently to reconcile CRC subtypes as a part of the CRCSC [11], with the difference that it weights sub-groups of a larger cluster as strongly as identical clusters of the same size (see Methods). Here, we applied the PMI method to reconcile subtypes from NMF, HC and KM similar to what we performed using the hypergeometric test. Unlike the hypergeometric method, PMI identified five communities (bPMI1 to 5; Fig. 3b and Additional file 3: Figure S3B), four (bPMI2 to 5) of which were analogous to hypergeometric communities (bHYP2, 3, 4 and 5). The final community (bPMI1) was a combination of the two basal hypergeometric communities (bHYP1 and 6). These communities were significantly associated with known subtypes, overall (Fisher's exact test; $p<0.001)$. As expected, four of the five communities represent luminal A (bPMI3 and 4), luminal B (bPMI2) and basal (bPMI1) communities (hypergeometric; $F D R<0.05$ ). The other community (bPMI5) was a mixture of HER2/ERBB2 and normal-like (Fig. 3b and Additional file 3: Figure S3B).

The merging of the bHYP1 and bHYP6 communities into bPMI1 is due to the large differences in scale of the edges connecting bHC1 to bNMF5/bKM4 and bHC1 to bNMF3/bKM1 in bHYP1 and bHYP6 versus bPMI1 (Fig. 3). FDR-corrected $p$-values between bHC1 and bNMF5/ bKM4 were 11-14 orders of magnitude larger than those between bHC1 and bNMF3/bKM1 (despite still being significant). Conversely, the weakest connection to bHC1 in bPMI1 is a PMI value of 0.64 , versus 0.77 for bNMF5 to bKM4 and 1.0 for bHC1 to bNMF3 and bHC1 to bKM1 (Fig. 3). This could indicate that the PMI method is able to detect the canonical basal subtype, while the hypergeometric reconciliation is able to find a sub-stratification of this subtype in this breast cancer data set.

To choose the optimal "subtype communities" between HYP and PMI communities, we calculated the silhouette width [26] for all samples in the different communities (Fig. 3 and Additional file 4: Figure S4). The average silhouette widths for HYP communities were 0.06 and that for PMI communities were 0.07 . Hence, PMI communities with highest average silhouette width were chosen as optimal.

This application of the pipeline to a well-characterised cancer has demonstrated its ability to identify new biologically distinct "subtype communities" of patients, alongside those subtypes which have already been extensively described. We next sought to apply this pipeline to a cancer with molecular subtypes that have not been explored so comprehensively, uveal melanoma - although classes at the gene expression level are known [35-37].

\section{Application to uveal melanoma and identification of novel "subtype communities" Identification of subtype communities}

Compared to breast cancer, uveal melanoma is a cancer type that has not been extensively subtyped, presumably due to its low incidence. This scarcity of samples makes clustering a challenge - clusters discovered are less likely to be robust due to their small size. It is in cases such as this where the reconciliation of clusters from multiple algorithms may present benefits in terms of increasing confidence in the results of clustering.

As with the breast cancer data, we applied the three clustering algorithms of $\mathrm{HC}, \mathrm{KM}$ and NMF to a dataset of the 6146 most variable genes $(\mathrm{SD}>0.8)$ from 58 patients with uveal melanoma (GSE22138, [22]). By performing the same assessment of cophenetic correlation coefficient, silhouette width and consensus matrices, we discovered four clusters by $\mathrm{HC}$, six clusters by $\mathrm{KM}$ and five clusters by NMF (Additional file 5: Figure S5A-D).

By reconciling these subtypes by a hypergeometric test followed by community detection, we identified five "subtype communities" of clusters (Fig. 4a). When we assessed these communities for the key molecular feature of chromosome 3 aneuploidy, we discovered a significant association of these communities with this feature (Fisher's exact test; $p<0.001$ ); one community melanoma mHYP2 - was significantly enriched (hypergeometric test; $F D R<0.001)$ for monosomy, and another (mHYP5) was significantly enriched $(F D R<0.05)$ for both disomy and partial monosomy (Fig. 4a and Additional file 6: Figure S6A). Two of the remaining three communities showed less significant associations with chromosome 3 disomy (mHYP4) and monosomy (mHYP1; hypergeometric test; $F D R<0.2$ ) respectively, while the final community (mHYP3) was not significantly enriched for either. A similar pattern of associations was observed when assessing four "subtype communities" defined by the PMI method (Fig. 4b), with one community each representing monosomy and disomy (mPMI1 and mPMI4, respectively), and one mixed disomy/partial monosomy/monosomy community (mPMI2) - however the association was not statistically significant (Fisher's exact test; $p=0.6$ ) (Fig. $4 \mathrm{~b}$ and Additional file 6: Figure S6B). HYP subtypes were chosen over PMI subtypes for significant association with known key molecular features of uveal melanoma and having a lower number of samples with negative silhouette width in this cohort (Additional file 7: Figure S7).

\section{Biological understanding of uveal melanoma subtype communities}

Next, we sought to understand these communities by performing GSEA, and discovered that one of these communities (mHYP1) was significantly enriched 

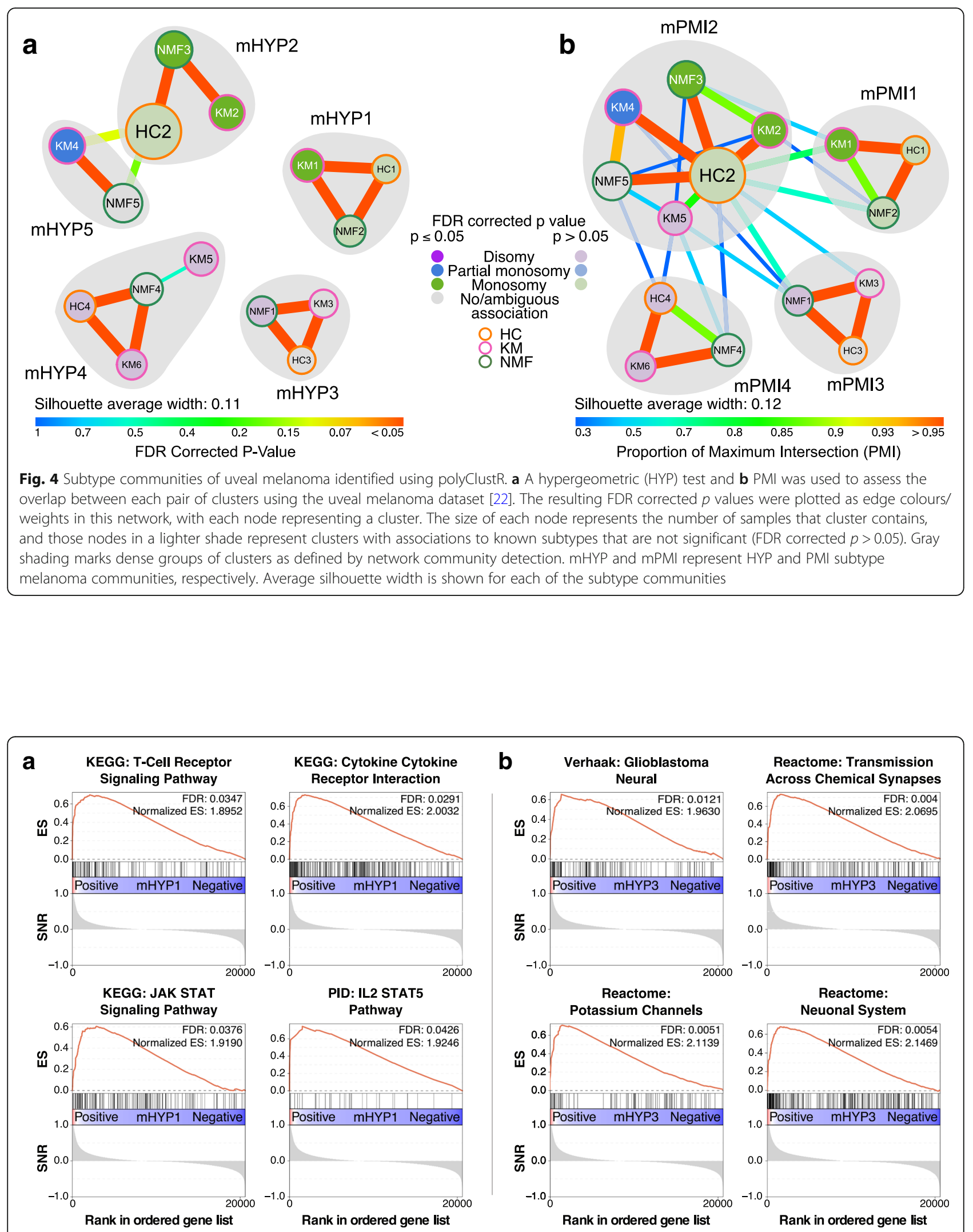

Fig. 5 GSEA enrichment plots of a the mHYP1 uveal melanoma community, showing significant enrichment of immunity-related gene sets, and b the mHYP3 uveal melanoma community, showing significant enrichment of neural-related gene sets. SNR - signal to noise ratio 
$(F D R<0.05)$ for gene sets associated with immune pathways (e.g. $\mathrm{T}$ cell receptor signaling, JAK-STAT, cytokine-cytokine receptor interactions, and IL2 STAT5 pathways; $F D R<0.05$; Fig. 5a). On the other hand, another subtype (mHYP3) was associated with neural cell types (e.g. glioblastoma neural subtype, neurotransmitter signaling, potassium signaling and neuron system; Fig. 5b; $F D R<0.05)$. The last communities (mHYP2, mHYP4 and mHYP5) did not significantly associate with any gene sets. This could indicate that mHYP2 (which is enriched for chromosome 3 monosomy) and mHYP4 (which is borderline enriched for chromosome 3 disomy) may be defined by their karyotype as opposed to a coherent transcriptomic pattern.

\section{Patient prognostic differences between uveal melanoma subtype communities}

Since more than $50 \%$ of uveal melanoma patients undergo metastasis [22], we assessed the metastasis-free prognosis of the uveal melanoma subtype communities using the GSE22138 [22] data set. Among the two highly frequent communities, mHYP2 (36\%) showed significantly poorer metastasis-free prognosis, whereas $\mathrm{MHYP} 5$ (27\%) showed better prognosis. Both mHYP4 (20\%) and mHYP1 (13\%) communities showed intermediate prognosis (Fig. 6a).

\section{Validation of uveal melanoma subtype communities}

Due to the low frequency of some of these communities in this dataset (5\% mHYP3, 13\% mHYP1), we sought to validate them in an independent dataset consisting of 58 patients with uveal melanoma (GSE44295). Patients were assigned to subtypes based on the correlation of their gene expression profile with the prediction analysis of microarrays (PAM) [38] centroids of each community. 57 samples had metastasis-free survival information. In the validation cohort, $32 \%$ of patients were assigned to the mHYP1 (immune-enriched) group, 19\% mHYP2 (monosomy-enriched), 12\% mHYP3 (neural-enriched), 5\% mHYP4 (undetermined) and 32\% mHYP5 (disomy/ partial monosomy-enriched). In terms of prognosis, these groups showed statistically significant differential metastasis-free survival ( $p=0.007$; Fig. 6b). Analogous to the previous dataset, mHYP2 and mHYP5 communities showed poor and good prognosis, respectively. While mHYP1 showed intermediate prognosis, mHYP4 couldn't be assessed due to low sample size of only $5 \%(n=3)$. Interestingly and similar to the training dataset (GSE22138), 82\% of mHYP2 (monosomy-enriched) group in the validation cohort underwent metastasis during follow-up, compared to only $11 \%$ of the mHYP5 (disomy/partial monosomy-enriched) group patients. In addition, 33\% of intermediate prognostic mHYP4 (undetermined)

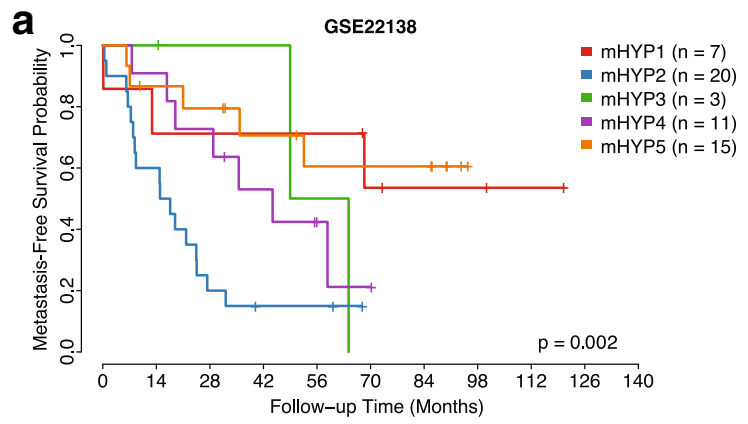

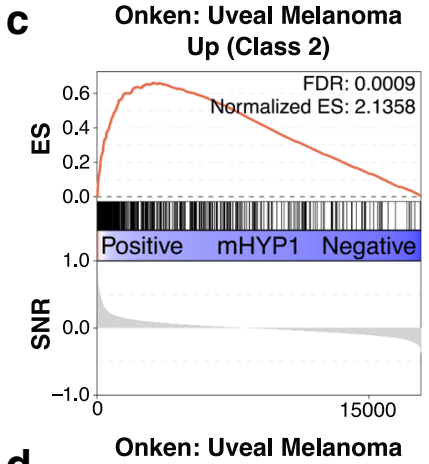

d

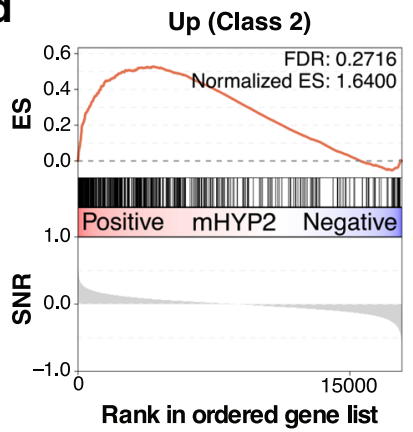

Fig. 6 Prognosis and GSEA analysis of uveal melanoma subtype communities. a-b Metastasis-free survival in the a discovery and $\mathbf{b}$ validation cohorts, respectively, was significantly different between communities. c-d GSEA enrichment plots of $\mathbf{c}$ the $\mathrm{mHYP} 1$ and $\mathbf{b}$ the mHYP2 $\mathrm{uveal}$ melanoma communities, showing significant enrichment of class 2 published subtypes. SNR - signal to noise ratio 
and $44 \%$ mixed prognostic mHYP1 (immune-enriched) patients experienced metastasis. With the increased sample size of mHYP3 (neural-enriched) community, we observed that it has poor overall survival and $57 \%$ of the mHYP3 samples underwent metastasis (Fig. 6b). Overall, this identifies and validates novel uveal melanoma subtype communities and their prognostic significance.

\section{Comparison of subtype communities to known uveal melanoma classes}

Previously, transcriptomic subtypes of uveal melanoma have been defined by clustering of gene expression profiles. Two classes were discovered - class 1, with good prognosis and association with chromosome 3 disomy; and class 2, with poor prognosis, associated with chromosome 3 monosomy and metastasis [35-37]. To reconcile these communities with the gene expression subtypes, we checked for gene set enrichment of the gene signatures for class 1 and class 2 uveal melanomas in this cohort [36]. The class 2 signature was enriched and borderline enriched in the mHYP1 community (immune-enriched; FDR < 0.001; Fig. 6c) and mHYP2 (monosomy; $\mathrm{FDR}=0.3$; Fig. $6 \mathrm{~d}$ ) groups, respectively, whereas, unexpectedly, the class 1 signature was not significantly enriched in any other group. This could indicate that the class 1 signature defines a heterogeneous set of patients who are not confined to any of our given communities. Overall, this analysis suggests that our novel uveal melanoma subtype communities reveal additional heterogeneity with clinical significance that requires further investigation.

\section{Conclusions}

These results demonstrate that no one clustering algorithm may be relied on to produce clusters which are robust and capture all heterogeneity in a dataset. Instead, multiple algorithms may be applied to the same dataset, and their results compared and reconciled. Our polyClustR tool provides a straightforward interface to cluster datasets using multiple algorithms, provides statistics on the quality of each clustering, and allows the user to fully understand how each result is related through multiple reconciliations. The demonstration that some low-frequency clusters - which may be lost or discarded as outliers if only one algorithm is applied are consistently identified across algorithms lends credence to their validity, and here such communities were additionally validated in an independent dataset. Thus, the reconciliation of multiple clustering results enables finer stratification of patients' molecular profiles enabling more focused biological profiling.

\section{Additional files}

\begin{abstract}
Additional file 1: Figure S1. Evaluation of consensus clustering of the breast cancer dataset from $k=2$ to $k=10$. (A) Cophenetic correlation coefficient (upper) and silhouette width (lower) of the clustering generated by each algorithm for each of $k$ clusters. (B) Consensus matrices for NMF clustering. Colors towards red indicate high consensus between runs and those towards blue indicate low consensus. (C-D) Consensus matrices for C) hierarchical clustering and D) k-means clustering. Blue indicates high consensus between different clustering runs and white indicates low consensus. (PDF $472 \mathrm{~kb}$ )
\end{abstract}

Additional file 2: Figure S2. Gene Set Enrichment Analysis (GSEA) analysis between subtypes in breast cancer. (A-F) GSEA analysis between the A) bNMF2 and B) bNMF6 breast cancer clusters, showing gene enrichment of metaplastic breast cancer and 17q21-25 amplicon signatures and C-F) between the two basal-subtype (bKM1 and bKM4) k-means clusters, showing enrichment of invasive and immune-related gene sets in bKM4 cluster. (PDF 119 kb)

Additional file 3: Figure S3. Comparison of community classifications from each reconciliation method with intrinsic breast cancer subtypes. (A-B) Heatmap showing hypergeometric test with overlap between the subtype communities (from polyClustR) and the known subtypes from A) hypergeometric and B) PMI reconciliation methods. Norm - normal-like subtype; LumA - luminal A subype; Lum B - luminal B subtype. (PDF 50 kb)

Additional file 4: Figure S4. Silhouette width of each sample and community in breast cancer for each reconciliation method hypergeometric (HYP; left) and PMI (right). Colors represent distinct subtype communities. (PDF $21 \mathrm{~kb}$ )

Additional file 5: Figure S5. Evaluation of consensus clustering of the uveal melanoma dataset from $k=2$ to $k=10$. (A) Cophenetic correlation coefficient (upper) and silhouette width (lower) of the clustering generated by each algorithm for each of $k$ clusters. (B) Consensus matrices for NMF clustering. Colors towards red indicate high consensus between different clustering runs and those towards blue indicate low consensus. (C-D) Consensus matrices for $C$ ) hierarchical clustering and D) k-means clustering. Blue indicates high consensus between clustering runs and white indicates low consensus. (PDF $467 \mathrm{~kb}$ )

Additional file 6: Figure S6. Comparison of community classifications from each reconciliation method with known chromosome 3 ploidy statuses in uveal melanoma. (A-B) Heatmap showing hypergeometric test with overlap between the subtype communities (from polyClustR) and the known ploidy status from A) hypergeometric and B) PMI reconciliation methods. (PDF 53 kb)

Additional file 7: Figure S7. Silhouette width of each sample and community in uveal melanoma for each reconciliation method hypergeometric (HYP; left) and PMI (right). Colors represent distinct subtype communities. (PDF $19 \mathrm{~kb}$ )

\section{Abbreviations}

CMS: Consensus molecular subtypes; CRC: Colorectal cancer; CRCSC: Colorectal cancer subtyping consortium; ER: Estrogen receptor; FDR: False discovery rate; GSEA: Gene set enrichment analysis; HC: Hierarchical clustering; KM: K-means (clustering); NMF: Non-negative matrix factorisation (clustering); PAM: Prediction analysis of microarrays; PMI: Proportion of maximum intersection; SD: Standard deviation

\section{Acknowledgments}

We thank Yatish Patil and Elisa Fontana for helping with critical assessment of certain results of the manuscript.

\section{Funding}

KE was in part funded, as a part of the Institute of Cancer Research (ICR)'s Undergraduate Vacation Scholarship Scheme, by the Engineering and Physical Sciences Research Council (EPSRC; grant no. EP/J500240/1) under the supervision of AS. We acknowledge NHS funding to the NIHR Biomedical Research Centre at The Royal Marsden NHS Foundation Trust and The Institute of Cancer Research, London, UK. 


\section{Availability of data and materials}

Gene expression data analysed in this study are publicly available from the original publications (breast cancer data [21] and uveal melanoma [22, 23]) and through the online source from the original paper - http://cancer.lbl.gov/ breastcancer/data.php (breast) and Gene Expression Omnibus (GEO) with accession numbers GSE22138 and GSE44295 (uveal melanoma).

\section{Authors' contributions}

KE developed the polyClustR package, performed all the experiments, analysed and interpreted the results, and wrote the manuscript. GN helped with the statistical methods and oversaw the data analysis. AS conceived the idea, oversaw the data analysis, interpreted the results and wrote the manuscript. All authors read and approved the final version of the manuscript.

\section{Ethics approval and consent to participate}

Not applicable.

\section{Competing interests}

AS has ownership interest as a patent inventor for a patent entitled "Colorectal cancer classification with different prognosis and personalized therapeutic responses" (patent number PCT/IB2013/060416). No potential conflicts of interest were disclosed by the other authors.

\section{Received: 10 December 2017 Accepted: 14 May 2018}

Published online: 25 May 2018

\section{References}

1. Hudis CA. Trastuzumab - mechanism of action and use in clinical practice. New Engl J Med. 2007;357:39-51.

2. Sadanandam A, Lyssiotis CA, Homicsko K, Collisson EA, Gibb WJ, Wullschleger S, Ostos LCG, Lannon WA, Grotzinger C, Del Rio M, et al. A colorectal cancer classification system that associates cellular phenotype and responses to therapy. Nat Med. 2013;19:619-25.

3. Song N, Pogue-Geile KL, Gavin PG, Yothers G, Rim Kim S, Johnson NL, Lipchick C, Allegra CJ, Petrelli NJ, O'Connell MJ, et al. Clinical outcome from oxaliplatin treatment in stage $\| / / I I$ colon cancer according to intrinsic subtypes: secondary analysis of NASBP C-07/NRG oncology randomized clinical trial. JAMA Oncol. 2016;2:1162-9.

4. Collisson EA, Sadanandam A, Olson P, Gibb WJ, Truitt M, Gu S, Cooc J, Weinkle J, Kim GE, Jakkula L. Subtypes of pancreatic ductal adenocarcinoma and their differing responses to therapy. Nat Med. 2011;17:500-3.

5. Moffitt RA, Marayati R, Flate EL, Volmar KE, Loeza SGH, Hoadley KA, Rashid $\mathrm{NU}$, Williams LA, Eaton SC, Chung AH, et al. Virtual microdissection identifies distinct tumor-and stroma-specific subtypes of pancreatic ductal adenocarcinoma. Nat Genet. 2015;47:1168-78.

6. Heiser LM, Sadanandam A, Kuo W-L, Benz SC, Goldstein TC, Ng S, Gibb WJ, Wang NJ, Ziyad S, Tong F, et al. Subtype and pathway specific responses to anticancer compounds in breast cancer. Proc Natl Acad Sci. 2012;109:2724-9.

7. Perou CM, Sørlie T, Eisen MB, van de Rijn M, Jeffrey SS, Rees CA, Pollack JR, Ross DT, Johnsen H, Akslen LA, et al. Molecular portraits of human breast tumours. Nature. 2000;406:747-52.

8. Wu L, Liu Z, Xu J, Chen M, Fang H, Tong W, Xiao W. NETBAGs: a networkbased clustering approach with gene signatures for cancer subtyping analysis. Biomark Med. 2015;9:1053-65.

9. Han J, Pei J, Kamber M. Data mining: concepts and techniques. 3rd ed. USA: Elsevier; 2011.

10. Sadanandam A, Wang X, de Sousa EMF, Gray JW, Vermeulen L, Hanahan D, Medema JP. Reconciliation of classification systems defining molecular subtypes of colorectal cancer: interrelationships and clinical implications. Cell Cycle. 2014;13:353-7.

11. Guinney J, Dienstmann R, Wang X, de Reyniès A, Schlicker A, Soneson C, Marisa L, Roepman P, Nyamundanda G, Angelino P, et al. The consensus molecular subtypes of colorectal cancer. Nat Med. 2015;21:1350-6.

12. Navarro JF, Frenk CS, White SDM. A universal density profile from. hierarchical clustering. Astrophys J. 1997:490:493.

13. Defays D. An efficient algorithm for a complete link method. Comput J, 1977;20:364-6.

14. MacQueen J. Some methods for classification and analysis of multivariate observations. Proceedings of the fifth Berkeley symposium on mathematical statistics and probability. 1967;1:281-97.
15. Lee DD, Seung HS. Learning the parts of objects by non-negative matrix factorization. Nature. 1999;401:788-91.

16. De Sousa E, Melo F, Wang X, Jansen M, Fessler E, Trinh A, de Rooij LPMH, de Jong $J$, de Boer OJ, van Leersum R, Bijlsma MF, et al. Poor-prognosis colon cancer is defined by a molecularly distinct subtype and develops from serrated precursor lesions. Nat Med. 2013;19:614-8.

17. Budinska E, Popovici V, Tejpar S, D'Ario G, Lapique N, Sikora KO, Di Narzo AF, Yan P, Graeme Hodgson J, Weinrich S, et al. Gene expression patterns unveil a new level of molecular heterogeneity in colorectal cancer. J Pathol. 2013;231:63-76.

18. Marisa L, de Reyniès A, Duval A, Selves J, Gaub MP, Vescovo L, EtienneGrimaldi MC, Schiappa R, Guenot D, Ayadi M, et al. Gene expression classification of colon cancer into molecular subtypes: characterization, validation, and prognostic value. PLoS Med. 2013;10:e1001453.

19. Roepman P, Schlicker A, Tabernero J, Majewski I, Tian S, Moreno V, Snel MH, Chresta CM, Rosenberg R, Nitsche U, et al. Colorectal cancer intrinsic subtypes predict chemotherapy benefit, deficient mismatch repair and epithelial-to-mesenchymal transition. Int J Cancer. 2013;134:552-62.

20. Schlicker A, Beran G, Chresta CM, McWalter G, Pritchard A, Weston S, Runswick S, Davenport S, Heathcote K, Castro DA, et al. Subtypes of primary colorectal tumors correlate with response to targeted treatment in colorectal cell lines. BMC Med Genet. 2012;5:1-15.

21. Chin K, DeVries S, Fridlyand J, Spellman PT, Roydasgupta R, Kuo WL, Lapuk A, Neve RM, Qian Z, Ryder T, et al. Genomic and transcriptional aberrations linked to breast cancer pathophysiologies. Cancer Cell. 2006;10:529-41.

22. Laurent C, Valet F, Planque N, Silveri L, Maacha S, Anezo O, Hupe P, Plancher C, Reyes C, Albaud B, et al. High PTP4A3 phosphatase expression correlates with metastatic risk in uveal melanoma patients. Cancer Res. 2011;71:666-74.

23. Triozzi PL, Achberger S, Aldrich W, Crabb JW, Saunthararajah Y, Singh AD. Association of tumor and plasma microRNA expression with tumor monosomy-3 in patients with uveal melanoma. Clin Epigenetics. 2016;8:80.

24. Field MG, Decatur $C L$, Kurtenbach $S$, Gezgin $G$, van der Velden PA, Jager MJ, Kozak KN, Harbour JW. PRAME as an independent biomarker for metastasis in uveal melanoma. Clin Cancer Res. 2016;22(5):1234-42.

25. Sokal RR, Rohlf FJ. The comparison of Dendrograms by objective methods. Taxon. 1962;11:33-40.

26. Rousseeuw PJ. Silhouettes: a graphical aid to the interpretation and validation of cluster analysis. J Comput Appl Math. 1987;20:53-65.

27. Raghavan UN, Albert R, Kumara S. Near linear time algorithm to detect community structures in large-scale networks. Phys Rev E. 2007;76:36106.

28. Subramanian A, Tamayo P, Mootha VK, Mukherjee S, Ebert BL, Gillette MA, Paulovich A, Pomeroy SL, Golub TR, Lander ES, et al. Gene set enrichment analysis: a knowledge-based approach for interpreting genome-wide expression profiles. Proc Natl Acad Sci. 2005;102:15545-50.

29. Wilkerson MD, Hayes DN. ConsensusClusterPlus: a class discovery tool with confidence assessments and item tracking. Bioinformatics. 2010;26:1572-3.

30. Gaujoux R, Seoighe C. A flexible R package for nonnegative matrix factorization. BMC Bioinformatics. 2010;11:367.

31. Csárdi G, Nepusz T. The igraph software package for complex network research. InterJournal Complex Systems. 1695;2006:1-9.

32. Maechler M, Rousseeuw P, Struyf A, Hubert M, Hornik K. Cluster: cluster analysis basics and extensions. In: R package version 2.0.4; 2016

33. Therneau T. A package for survival analysis in $S$. In: R package version $2.37-4 ; 2014$

34. Sorlie T, Tibshirani R, Parker J, Hastie T, Marron JS, Nobel A, Deng S, Johnsen $H$, Pesich R, Geisler S, et al. Repeated observation of breast tumor subtypes in independent gene expression data sets. Proc Natl Acad Sci U S A. 2003; 100(14):8418-23.

35. Onken MD, La W, Ehlers JP, Harbour JW. Gene expression profiling in uveal melanoma reveals two molecular classes and predicts metastatic death advances in brief gene expression profiling in uveal melanoma reveals two molecular classes and predicts metastatic death. Cancer Res. 2004;64(20):7205-9.

36. Onken MD, Worley LA, Dávila RM, Char DH, Harbour JW. Prognostic testing in uveal melanoma by transcriptomic profiling of fine needle biopsy specimens. J Mol Diagn. 2006;8:567-73.

37. Worley LA, Onken MD, Person E, Robirds D, Branson J, Char DH, Perry A, Harbour JW. Transcriptomic versus chromosomal prognostic markers and clinical outcome in uveal melanoma. Clin Cancer Res. 2007;13:1466-71.

38. Tibshirani R, Hastie T, Narasimhan B, Chu G. Diagnosis of multiple cancer types by shrunken centroids of gene expression. Proc Natl Acad Sci. 2002; 99:6567-72 